\title{
Individual and Population Sex Allocation Patterns*
}

\author{
STEVFN A. Frank \\ Division of Biological Sciences, University of Michigan, \\ Ann Arbor, Michigan 48109-1048 \\ Received April 30, 1985
}

\begin{abstract}
A variety of sex allocation models is considered in which (i) the reproductive returns on investment in males differ from the returns on investment in females, (ii) the amounts of resources available for reproduction vary in the population, (iii) the costs of making male and female reproductive structures differ, and (iv) the conception sex ratio may be fixed and there may be an initial minimum investment per offspring. Results of these models include quantatitive predictions for both individual- and population-level sex allocation, an opportunity to study the magnitude of changes in predicted patterns as key variables change, and therefore an analysis of the robustness of Fisher's equal investment theory. One example is that Fisher's argument is extremely robust for high fecundity organisms, but, in low fecundity organisms, is sensitive to differences between the sexes in reproductive returns on investment per offspring, a situation that occurs in many vertebrates to which Fisher's theory is often applied. A second example is that individual- and population-level patterns often depend strongly on the distribution of resources available for reproduction among individuals in the population. $\quad 1987$ Academic Press, Inc.
\end{abstract}

\section{INTRODUCTION}

Fisher (1930) asserted that equal amounts of resources will be allocated to sons and daughters within a population in each generation, since any asymmetry favors more investment in the under-endowed sex. Fisher's idea continues to be the cornerstone of sex allocation theory, although very simple results prove the equal investment theory to be sensitive to changes in a crucial and often unrealistic assumption. This critical assumption is that reproductive returns on investment must be the same for resources allocated to male or female reproductive function (MacArthur, 1965; Hamilton, 1967; Clark, 1978; Charnov, 1979a; Charlesworth and Charlesworth, 1981). Charnov (1982) has reviewed many cases in which unequal investment results from different returns on investment for the two sexes.

* Research supported by NIH Grants 1-T32-GM-07544-07 and R01-GM-32589. 
The current theory for different returns between the sexes deals only with special cases. Also, no general approach exists for predicting individual and population sex allocations when there is variation among individuals in resources available for reproduction. Here I subsume and extend previous work with a simple and general formulation, which yields more realistic quantitative predictions. Three new outcomes of this general approach are that individual and population sex allocations often depend strongly and in a non-intuitive way on the shape of the distribution of resources among individuals, that the robustness of Fisher's equal investment theory can be analyzed in a wide variety of situations, and that several remaining weaknesses of the current theory can be made explicit.

I begin by deriving a general result for the population allocation ratio from the shapes of the male and female return functions (see Charnov, 1979a; Charlcsworth and Charlcsworth, 1981 for special cases), and then present a general solution for the case in which individuals have different amounts of resources to invest, and can adjust their allocation ratios according to the resources available to them. These problems will first be considered with total reproductive returns as a function of total investment in males and females per investment interval, where individuals may have different amounts of resources available to invest. I then consider the problems that arise when individuals can vary the amount of investment per offspring (i.e., how to package investment into discrete units), and also when the sex ratio at conception is fixed (Maynard Smith, 1980), or when there is a fixed minimum investment per sex (Heath, 1977). In the discussion 1 use the insight generated by this group of models to consider how the problem of defining an "investment period" affects predicted allocation patterns, and when it is necessary to analyze investment per offspring versus total investment per sex.

For any particular case the models here allow one either to examine the magnitude of changes in predicted patterns as functions of changes in key variables, or else they suggest ways in which to construct appropriate models. The work presented here is based on a number of separate studies of narrow special cases which will be referred to later. As far as I know, this is the first attempt to generalize these separate problems within a formal quantitative structure.

I will assume autosomal control of the sex ratio and additive gene action, and will often present the argument in the form of the mother's reproductive success as a function of her allocation ratio in sons and daughters. The syntheses of Charnov (1982) and Bull (1983) show that there are only a few minor differences in the argument, if any at all, when the organism is hermaphroditic, when sex (gender) is environmentally determined, or when the father partly or completely controls the allocation ratio. 


\subsection{Review of Sex Allocation Algebra}

The models will be constructed by searching for the sex allocation phenotype that maximizes the relative reproductive success of the individual that controls the allocation ratio. This approach is common in the sex ratio literature, originating with Shaw and Mohler (1953). Charnov (1982) reviews the history of this approach for sex ratios, and Maynard Smith (1982) and Grafen (1984) discuss the relationship between phenotypic (ESS) and genetic models. Taylor (1985) compares the Shaw-Mohler approach with other mathematical methods for the study of sex allocation. By making four assumptions, results derived by the phenotypic method are usually the same as results obtained from genetic models. These assumptions are (reviewed by Charnov, 1982) (i) large population size, (ii) random mating, (iii) no within-sex competition for a limited resource, and (iv) control of the sex allocation ratio by additive gene action at a single autosomal locus in a diploid organism. See Uyenoyama and Bengtsson $(1979,1981,1982)$, Eshel and Feldman (1982a, b), and Taylor (1985), for further discussion of genetic and phenotypic sex ratio models.

Assume that mothers control the allocation of resources to sons and daughters. The expected relative reproductive success for the $i$ th mother of a large population can be written as a function of the fraction of resources allocated to sons, $x_{i}$, and the fraction allocated to daughters, $1-x_{i}$ (Shaw and Mohler, 1953; MacArthur, 1965). Let the reproductive returns on male investment be $f\left(x_{i}\right)$, and returns on female investment be $g\left(1-x_{i}\right)$. Then the relative reproductive value of malc investment for the $i$ th mother is $f\left(x_{i}\right) / E\left[f\left(x_{i}\right)\right]$, where the denominator is the expectation over all mothers in the population. Likewise, relative returns on female investment are $g\left(1-x_{i}\right) / E\left[g\left(1-x_{i}\right)\right]$. So, relative reproductive success of the $i$ th mother is

$$
w_{i}=\frac{f\left(x_{i}\right)}{E\left[f\left(x_{i}\right)\right]}+\frac{g\left(1-x_{i}\right)}{E\left[g\left(1-x_{i}\right)\right]}
$$

or

$$
w_{i}=f\left(x_{i}\right)\left(\frac{E\left[g\left(1-x_{i}\right)\right]}{E\left[f\left(x_{i}\right)\right]}\right)+g\left(1-x_{i}\right)
$$

Equation (1) is the standard form of the Shaw-Mohler (1953) equation, where population fitness $E\left(w_{i}\right)$ is always 2, independent of the individual allocation ratios $\left(x_{i}: 1-x_{i}\right)$. Relative fitness of the $i$ th mother in the population is the same for Eqs. (1) and (2), but $E\left(w_{i}\right)$ for (2) is $2 E\left[g\left(1-x_{i}\right)\right]$, which is sometimes useful, since population fitness will usually depend on the total reproductive value of the females in the population, when there is 
no paternal investment except gametes. Equation (2) is also useful, since it defines reproductive returns for male investment as value of sons, $f\left(x_{i}\right)$, times average mating success, $E(g) / E(f)$, and this allows reproductive returns for males and females to be compared on the same scale ( $f$ and $g$ have arbitrary scales and are not generally comparable). This standardization to a common metric will be used to generate comparisons of male and female return curves on the same set of axes.

If an equilibrium exists, it will occur at the values of $x_{i}$ and $1-x_{i}$ that maximize $w_{i}$ (MacArthur, 1965). There are several equivalent ways of going about this, and I have chosen a method that is useful for models developed below. The maximum of $w_{i}$ occurs where $f\left(x_{i}\right) / E\left[f\left(x_{i}\right)\right]$ is a maximum with respect to $x_{i}$ and simultaneously $g\left(1-x_{i}\right) / E\left[g\left(1-x_{i}\right)\right]$ is a maximum with respect to $1-x_{i}$, subject to the obvious constraint that $x_{i}+\left(1-x_{i}\right)=1$. Following through one obtains MacArthur's (1965) general result

$$
\frac{f^{\prime}\left(x^{*}\right)}{f\left(x^{*}\right)}=\frac{g^{\prime}\left(1-x^{*}\right)}{g\left(1-x^{*}\right)}
$$

which is a standard sort of marginal value result from classical economic theory. When returns are linear for both sons and daughters, $f\left(x^{*}\right)=x^{*}$ and $g\left(1-x^{*}\right)=1-x^{*}$, hence $x^{*}=\frac{1}{2}$. Here all individuals (i) have the same amount of energy to invest and (ii) all invest $x^{*}$ in males at equilibrium, so individual- and population-level investment is the same. These two assumptions will be relaxed later.

\subsection{Generating Shapes for the Return Functions}

There is no particular reason why the returns on male and female investment must be either linear or equal. The three basic shapes shown in Fig. 1 describe other likely sorts of return functions. For example, the

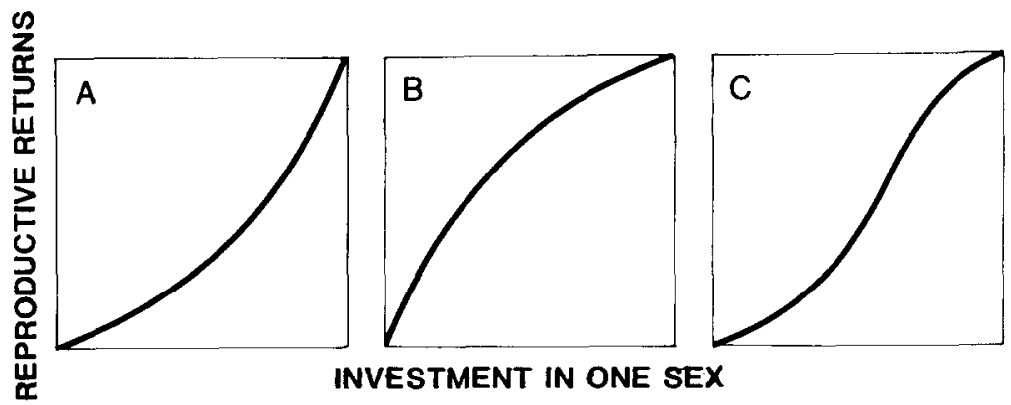

FIG. 1. Shapes for reproductive returns for one sex as a function of investment in that sex. $A$, low investment on one sex yields relatively low reproductive returns for that sex, while in $B$ low investment yields relatively high returns. An example of $S$-shaped returns, as in $C$, would be investment in males in a sexually dimorphic polygynous ungulate. 
diminishing returns in Fig. 1B describe the value of investment in sons when there is local competition for mates (Hamilton, 1967). The $S$-shaped curve in Fig. 1C describes the return function on male investment for most low fecundity polygynous species, since weak males rarely reproduce while strong males often sire several broods. Additional biological interpretations of return function shapes for each sex can be found in Charlesworth and Charlesworth (1981) and Charnov (1982).

Ideally, the form of the return function would allow $x^{*}$ to be calculated directly for any of these shapes. The cumulative distribution function (CDF) of the beta probability density function (PDF) has this generality. The CDF for a beta distribution is

$$
\begin{aligned}
& f(x)=\frac{\Gamma(s+t)}{\Gamma(s) \Gamma(t)} \int_{0}^{x} y^{s-1}(1-y)^{t-1} d y, \quad s, t>0,0 \leqslant x \leqslant 1 \\
& \Gamma(r)=(r-1) \Gamma(r-1)=\int_{0}^{x} y^{r} e^{1}{ }^{y} d y,
\end{aligned}
$$

where $\Gamma(r)$ is the gamma function, sometimes referred to as the "generalized factorial function," since if $r$ is a positive integer, $\Gamma(r)=(r-1) ! . s$ and $t$ are parameters that determine the shape of the function (Fig, 2).

\section{Population Allocation under Nonlinear Returns}

\subsection{Nonlinear Returns on Male Investment}

A model is studied in this section in which the shapes of the male and female return functions differ, each individual in the population has the

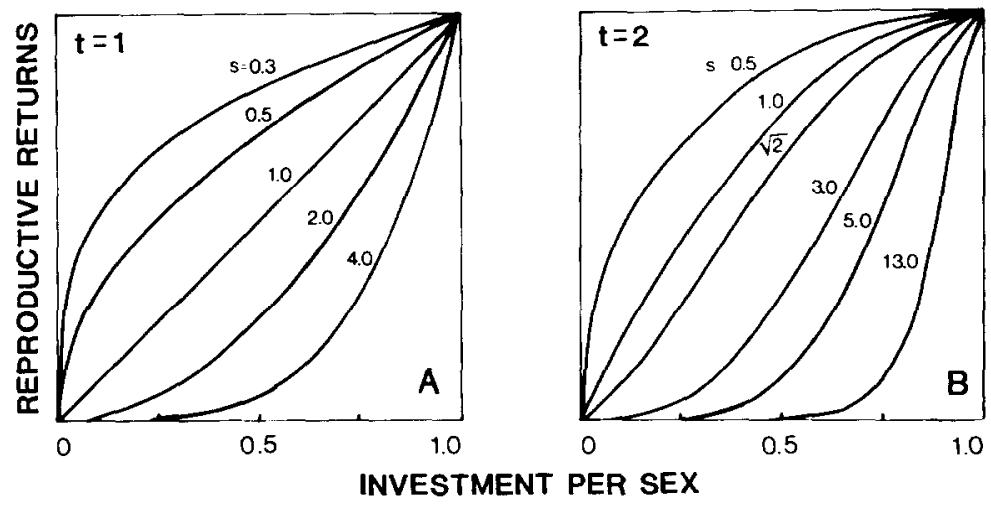

FiG. 2. Shapes for reproductive returns described by the parameters $s$ and $t$, from Eq. (4). 
same amount of resources to invest, and each is constrained to invest the same proportion of resources into males and females. Preventing variation among individuals makes this model unrealistic, but there are two reasons for beginning with this example. First, this is the only case for which a reasonably complete study has been made of population allocation under differing return functions (Charnov, 1979a, b), so beginning with this situation allows us to put the present paper into the context of previous work. Second, the development of more realistic models is made easier by beginning with a simple and familiar case, and then relaxing offending assumptions sequentially.

The effect of nonlinear returns on the allocation ratio can be illustrated most easily by assuming linear returns for one sex, and nonlinear returns for the other, so let $g\left(1-x_{i}\right)=1-x_{i}$; i.e., reproductive returns increase linearly with female investment. (Cases in which returns are nonlinear for both sexes can be handled in a straightforward, although more tedious, manner. See the Appendix.)

$f^{\prime}\left(x^{*}\right)$ can be written directly from Eq. (4), since it is the PDF of the beta function

$$
f^{\prime}\left(x^{*}\right)=\frac{d f\left(x^{*}\right)}{d x^{*}}=\frac{\Gamma(s+t)}{\Gamma(s) \Gamma(t)}\left(x^{*}\right)^{s-1}\left(1-x^{*}\right)^{t} \quad \text { ' }
$$

Substituting into Eq. (3),

$$
\frac{\left(x^{*}\right)^{s} \cdot{ }^{1}\left(1-x^{*}\right)^{t} \quad 1}{\int_{0}^{x^{*}} y^{\prime \prime}}=\frac{1}{1-y)^{\prime-1} d y}, \quad s, t>0 .
$$

When both $s$ and $t$ are not integers, the algebra is messy but tractable. For simplicity, $t$ will be constrained to integer values, since a rich array of shapes can be generated for these values of $t$, sufficient to describe nearly all realistic situations. If $t$ is a positive integer, the binomial expansion can be used to simplify $\left(1-x^{*}\right)^{t-1}$ and $(1-y)^{t} \cdot 1$. For example,

$$
(1-y)^{t} \quad{ }^{\prime}=\sum_{i=0}^{1}(-1)^{i} y^{i}, \quad t=1,2,3, \ldots
$$

Using the binomial expansion, Eq. (6) can be written as a polynomial in $x^{*}$

$$
\begin{aligned}
& \left\{\sum_{i=1}^{t}(-1)^{i}\left(x^{*}\right)^{i}\left(\begin{array}{c}
t-1 \\
i-1
\end{array}\right)\left(\frac{i+t(i+s-1)}{i(i+s-1)}\right)\right\}+1=0 \\
& s>0, \quad t=1,2,3, \ldots, \quad 0 \leqslant x^{*} \leqslant 1 \\
& \left(\begin{array}{c}
t-1 \\
i-1
\end{array}\right)=\frac{(t-1) !}{(t-1) !(i-1) !} \text {. }
\end{aligned}
$$


Local stability of the equilibrium against invasion by a rare mutant can be checked with the second derivative, yielding the conditions:

$$
\begin{array}{ll}
\text { (i) } s+t>2 ; s \neq 1 ; t \neq 1, & x^{*}>\frac{s-1}{s+t-2} \\
\text { (ii) } s+t<2 ; s \neq 1 ; t \neq 1, & x^{*}<\frac{s-1}{s+t-2} \\
\text { (iii) } s=1, & t>1 \\
\text { (iv) } t=1 & s<1 .
\end{array}
$$

In all four cases, $x^{*}$ is a global maximum over the interval $[0,1]$ if and only if $x^{*}<\frac{1}{2}$, and is a local, but not global, maximum when $x^{*}>\frac{1}{2}$. These results will be illustrated for the cases $t=1,2$. Charnov (1979a) has also developed a complete study of the case $t=1$. When $t=1$ and $s<1$, $x^{*}=s /(s+1)$ (Charnov, 1979a). Shapes of the return function on male investment are shown in Fig. 2A.

In general the equilibrium occurs where the slope of the tangent to the returns on male investment, $f^{\prime}\left(x^{*}\right)\left[g\left(1-x^{*}\right) / f\left(x^{*}\right)\right]$ (male returns include expected breeding success, $E(g) / E(f))$, is the same as the slope of the tangent to returns on female investment, $g^{\prime}\left(1-x^{*}\right)$ [see Eq. (3)]. In the cases examined here both of these slopes are 1 , since $g(1-x)=1-x$ and therefore $g^{\prime}=1$.

When $t=2$, we obtain the equilibrium

$$
x^{*}=\frac{(2 s+1)(s+1)-\sqrt{(5 s+1)(s+1)}}{2 s(s+2)}
$$

which is a global maximum for $s<\sqrt{2}$ (i.e., whenever $x^{*}<\frac{1}{2}$ ), and is a local, but not global, maximum for $\sqrt{2}<s<3$. Shapes for the return functions on male investment are presented in Fig. 2. When $s<\sqrt{2}$, and $x^{*}$ is a global maximum, the equilibrium again occurs where the tangent to the male return function has the same slope as the linear returns on female investment.

When $s>\sqrt{2}$, no monomorphic equilibrium exists. A full analysis of this region under a variety of assumptions about genetic control might prove interesting.

\subsection{Nonlinear Returns on Female Investment}

All the above results can also be applied when the return function for males and females are switched by replacing $1-x^{*}$ with $z^{*}$, and $x^{*}$ with 
$1-z^{*}$. Assuming returns on female investment are nonlinear and returns on male investment are linear, and substituting into Eq. (3), one obtains,

$$
\frac{1}{1-z^{*}}=\frac{g^{\prime}\left(z^{*}\right)}{g\left(z^{*}\right)}
$$

which is analogous to Eq. (6). For example, if $g\left(z^{*}\right)$ is the CDF of a beta distribution [see Eq. (4)] with parameters $t=1, s<1$, then $z^{*}=1-x^{*}=s /(s+1)$.

\section{Allocation when Resources Vary among Individuals}

The above models assume (i) that each individual has the same amount of resources to invest and (ii) that at equilibrium cvery individual will invest the same proportion of resources in males and females. These two assumptions are clearly unrealistic. Trivers and Willard (1973) were the first to note that in polygynous mammalian species, returns on male investment differ from returns on female investment, and that this may affect sex ratio patterns.

To fix ideas, consider the example of parasitic wasps discussed in detail by Charnov et al. (1981). A female Lariophagus lays a single egg on each weevil larva that it encounters. The size of the larva is probably a good measure of the amount of resources available to the mother in each independent investment period, since offspring size is associated with larval host size. This allows us to equate in a realistic way total investment per independent investment period with investment per offspring. In general, the model developed in this section applies only to total investment per investment period. A model that considers both investment per individual and total investment per period is developed later, and the difficulties that may arise when considering how to define properly independent investment pcriods will also be discussed.

Returning to this wasp example, there is reason to believe that female fecundity increases with size faster than does the expected number of matings increase with size for males (Charnov et al., 1981). Hence we know that there is a measurable resource distribution size of hosts - and that the shapes of the return functions differ between the sexes. Charnov et al. (1981) found that the wasps adjust their sex ratio according to the relative sizes of the hosts available: on relatively large hosts the wasps tended to produce more daughters, and on relatively small hosts they tended to produce more sons. It was also shown that a host of particular size may be treated as large if most other hosts are smaller, or small if most other hosts are larger. Charnov et al. (1981) analyze their data in light of the qualitative predictions of the Trivers-Willard theory. 
No general quantitative models exist that predict both the individual and population allocation ratios, given the distribution of resources among individuals and the shapes of the return functions for male and female investment, although Charnov (1979b) and Bull (1981) have described some unrealistically simple cases, and Charnov et al. (1981) indicated qualitatively how the problem might be approached. A general quantitative model is readily obtained by extending the techniques used in Section 2. The main value of such a model is that it allows one to explore the magnitude of predicted individual and population sex allocation changes as functions of changes in resource distribution and return curve shapes.

Let the $i$ th individual in the population have $k_{i}$ units of resources to invest, and let the units be standardized so that $0<k_{i} \leqslant 1$ for all $i$. As before, let $x_{i}$ be the proportion of resources that the $i$ th individual allocates to males, and $1-x_{i}$ be the proportion allocated to females. Then the ith individual invests $k_{i} x_{i}$ units of resources in males, and $k_{i}\left(1-x_{i}\right)$ units in females, and the relative fitness $w_{i}$ can be written as

$$
w_{i}=\frac{f\left(k_{i} x_{i}\right)}{E\left[f\left(k_{i} x_{i}\right)\right]}+\frac{g\left[k_{i}\left(1-x_{i}\right)\right]}{E\left(g\left[k_{i}\left(1-x_{i}\right)\right]\right)} .
$$

Maximizing $w_{i}$ with respect to $k_{i}, x_{i}$ and $k_{i}\left(1-x_{i}\right)$ yields

$$
\frac{f^{\prime}\left(k_{i} x_{i}^{*}\right)}{E\left[f\left(k_{i} x_{i}^{*}\right)\right]}=\frac{g^{\prime}\left[k_{i}\left(1-x_{i}^{*}\right)\right]}{E\left(g\left[k_{i}\left(1-x_{i}^{*}\right)\right]\right)} \quad \text { for all } i \text {. }
$$

It will again be assumed that returns are linear for female investment; and as before, the roles of males and females can be switched. With linear returns for females, $g=k_{i}\left(1-x^{*}\right)$ and $g^{\prime}=1$, so Eq. (12) becomes

$$
f^{\prime}\left(k_{i} x_{i}{ }^{*}\right) \frac{E\left[k_{i}\left(1-x_{i}^{*}\right)\right]}{E\left[f\left(k_{i} x_{i}^{*}\right)\right]}=1 \quad \text { for all } i \text {. }
$$

Once again, the equilibrium occurs where the slope of the tangent to the returns on male investment, $f^{\prime}[E(g) / E(f)]$, equals the slope of the tangent to the returns on female investment, here $g^{\prime}=1$. To obtain a solution for particular assumptions, we nced to specify a probability (frequency) distribution for the amount of resources, $k_{i}$, available to each individual. It is again convenient to use a beta distribution to describe shapes, in this case for the shapes of the frequency distribution (PDF) of resources rather than for the reproductive return curves described by the CDF. Let $k_{i}$ be the PDF of a beta distribution with parameters $a, b>0$.

$$
h\left(k_{i}\right)=\operatorname{Prob}\left(k_{i}=y\right)=\frac{\Gamma(a+b)}{\Gamma(a) \Gamma(b)} y^{a-1}(1-y)^{b-1}, \quad 0 \leqslant k_{i} \leqslant 1 .
$$

The mode of this distribution is at $(a-1) /(a+b-2)$. See Fig. 3 for shapes. 


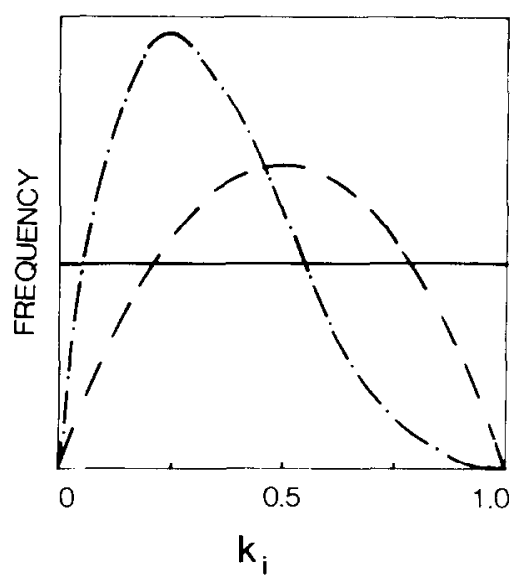

FIG. 3. Frequency distributions for amount of resources per individual, $k_{i}$, described by parameters $a$ and $b$; solid curve, $a=b=1$; dashed curve, $a=b=2$; dot-dash curve, $a=2$, $h=4$. The mode is at $(a-1) /(a+h-2)$. See Fq. (14).

I will consider separately the three shapes for the male return function (Figs. 1A-C). Let $f\left(k_{i} x_{i}\right)$ be the CDF of a beta distribution, as in Eq. (4), and with $t=1$ and $s>1$ (Fig. 2A). This gives the shape with an increasing slope shown in Fig. 1A. From the shape of the male return function, a reasonable conjecture is that at equilibrium, the set $\left\{x_{i}{ }^{*}\right\}$ is such that

$$
k_{i} x_{i}^{*}=\left\{\begin{array}{ll}
0 & k_{i}<\lambda \\
k_{i} & k_{i}>\lambda,
\end{array} \quad 0 \leqslant i \leqslant 1,\right.
$$

or that an individual produces all females when its resource level is less than $\lambda$, and all males if available resources are greater than $\lambda$, as suggested by Charnov (1979b) and Bull (1981). The population allocation ratio, $x^{*}$, for a large population is

$$
\begin{aligned}
x^{*} & =E\left(k_{i} x_{i}\right) / E\left(k_{i}\right) \\
& =\left(\int_{i}^{1} k_{i} h\left(k_{i}\right) d k_{i}\right) / E\left(k_{i}\right) .
\end{aligned}
$$

Individual fitness, $w_{i}$, from Eq. (2) is now

$$
w_{i}\left(k_{i} x_{i}\right)=f\left(k_{i} x_{i}\right) \frac{E\left[k_{i}\left(1-x_{i}\right)\right]}{E\left[f\left(k_{i} x_{i}\right)\right]}+k_{i}\left(1-x_{i}\right) .
$$

At equilibrium, for each $i$,

$$
w_{i}\left(k_{i} x_{i}^{*}\right) \geqslant w_{i}\left(k_{i} x_{j}\right) \quad \text { for all } x_{j} \neq x_{i}{ }^{*}
$$




$$
w_{i}\left(k_{i} x_{i}\right)= \begin{cases}k_{i}, & k_{i}<\lambda \\ \frac{f\left(k_{i}\right) E\left[k_{i}\left(1-x_{i}\right)\right]}{E\left[f\left(k_{i} x_{i}\right)\right]}, & k_{i}>\lambda \\ k_{i}=\frac{f\left(k_{i}\right) E\left[k_{i}\left(1-x_{i}\right)\right]}{E\left[f\left(k_{i} x_{i}\right)\right]}, & k_{i}=\lambda .\end{cases}
$$

Equation (19c) can be used to solve for $i$ for any distribution $f$ that is concave up (Fig. 1A). Here I continue to illustrate the general results with beta distributions, in order to gain some feeling for the quantitative effects of particular assumptions.

To solve, we need to specify the shape of the resource distribution function, $h\left(k_{i}\right)$. I illustrate the method first for $a=b=1$ (the uniform distribution), and then for $a=b=2$ (see Fig. 3). Since $t=1$, we have from Eq. (4), $f\left(k_{i} x_{i}\right)=\left(k_{i} x_{i}\right)^{y} / s$, and since $a=b=1, h\left(k_{i}\right)=1$, so

$$
\begin{aligned}
E\left[\left(k_{i} x_{i}\right)^{s}\right] & =\int_{0}^{1}\left(k_{i} x_{i}\right)^{s} h\left(k_{i}\right) d k_{i}=\int_{i}^{1}\left(k_{i}\right)^{s} d k_{i} \\
& =\frac{1 \lambda^{s+1}}{s+1} .
\end{aligned}
$$

Substituting into Eq. (19c) yiclds

$$
\dot{\lambda}=\frac{\left(\lambda^{s} / s\right)\left(i^{2} / 2\right)}{\left(1-\lambda^{s+1}\right) /[s(s+1)]} .
$$

Solving,

$$
\lambda=\left(\frac{2}{s+3}\right)^{1(s+1)}, \quad s>1
$$

So, when $k_{i}<\lambda, x_{i}{ }^{*}=0$, and when $k_{i}>\lambda, x_{i}{ }^{*}=1$. The population allocation ratio is, from Eq. (16), $x^{*}=1-\lambda^{2}$. These results are presented in Fig. 4.

When $k_{i}$ has the distribution defined by $a=b=2$ (Fig. 3 ), the result is shown in Fig. 4, and takes the form

$$
3(s+2)(s+7) \lambda^{s+3}-4(s+5)(s+3) \lambda^{s+2}+12=0 .
$$

Note that both $x^{*}$ and $\lambda$ change as the shape of the resource distribution curve changes, even when the resource curves have the same mean and median, as for $a=b=1$ versus $a=b=2$ (Fig. 4).

For the case $t=1, s<1$, there are diminishing returns on male investment (Figs. 1B and 2B). From Eq. (13) it can be seen that if $f^{\prime}\left(k_{i} x_{i}{ }^{*}\right)$ 

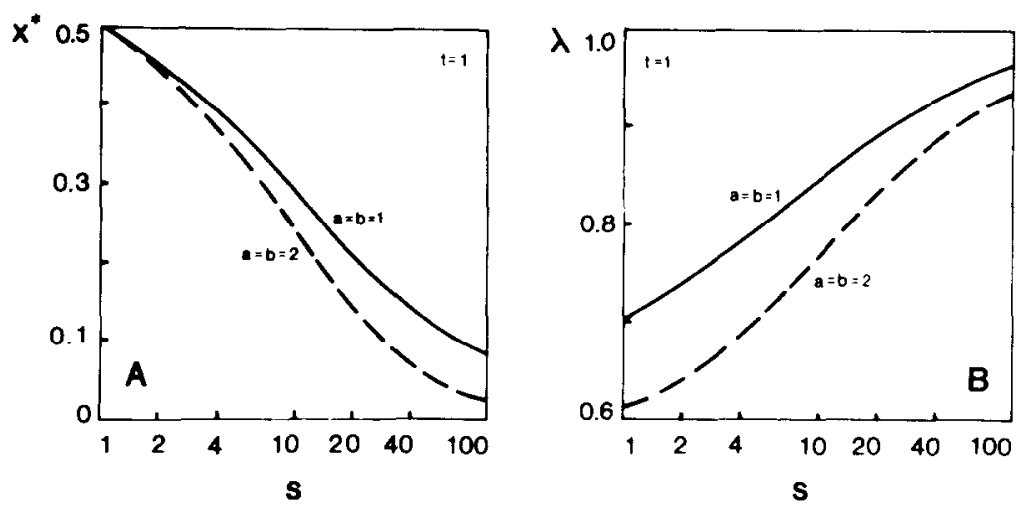

FIG. 4. Population and individual sex allocations when resources vary among individuals, with $t=1$. $A$, the stable population ratio, $x^{*}$, as a function of the shape of the resource distribution curve described by $a$ and $b$, and the shape of the reproductive returns curve on male investment, described by $s$ and $t . B$, the point of sex change, $\lambda$, where individuals with $k_{i}<i$ invest entirely in daughters, and with $k_{i}>\lambda$ invest entirely in sons. Note that both $x^{*}$ and $\lambda$ depend on the shape of the resource distribution curve, even when different distributions are symmetric and have the same mean and median, as with $a=b=1$ versus $a=b=2$.

is uniquely invertible, then $k_{i} x_{i}{ }^{*}=k_{j} x_{j}{ }^{*}$ for all $i, j$. The slope of $f\left(k_{i} x_{i}{ }^{*}\right)$ is monotone decreasing for $t=1, s<1$, so $f^{\prime}$ is uniquely invertible. If $K$ is defined as the expected value of $k_{i}$, and we impose the restriction $K x_{i} \leqslant k_{i} \leqslant 1$ for all $i$, then $k_{i} x_{i}=K x^{*}$ for all $i$, and substituting into Eq. (13), $x^{*}=s /(s+1)$, which is the same population allocation ratio as when $k_{i}=1$ for all $i$ (see above). It can be shown that when $k_{i}$ has a symmetric beta distribution, i.e., $a=b$, then $s /(2+s) \leqslant k_{i} \leqslant 1$ is the necessary restriction on $k_{i}$, and $K=(s+1) /(s+2)$. These results are easily interpreted: at $K x^{*}$ the tangent to the male return curve has the same slope as the linear returns on female investment, and the rate (slope) of increasing returns on male investment up to $K x^{*}$ are greater than for the constant rate for females, while the rate of increasing returns beyond $K x^{*}$ are less than for females. So the $i$ th individual invests $K x^{*}$ in males, and $k_{i}-K x^{*}$ in females. If an individual is constrained to produce either all males or all females $\left(x_{i}\right.$ is 0 or 1$)$, then the population average result is the same as for the first case above, and Eqs. (21) and (22) apply. Such a constraint would result, for example, when gender is environmentally determined (Bull, 1983), so that an individual is predicted to become either a male or female depending on the relative amount of resources it has to invest in reproduction.

The fact that individuals are favored to produce a mixture of males and females when the slope of the return curve for one sex is monotonically decreasing (Fig. 1B) relative to linear returns for the other sex is analogous 
to the result of Charnov et al. (1976) that this combination of shapes will favor hermaphroditism, i.e., a mixture of male and female investment.

The third and final case is where returns on male investment are described by an $S$-shaped curve (Fig. 1C). To illustrate, a family of $S$-shaped curves can be generated by setting $t=2$, for all $s \geqslant 3$ (Fig. 2B). An approximate solution can be obtained by following through the steps in Equations (15)-(19). The result is

$$
\begin{aligned}
& s(s+2)(s+3)(3 s+24) \lambda^{s+4} \\
& -(s+2)(s+4)[12(2 s+1)+(7 s+3)(s+3)] \lambda^{s+3} \\
& +(s+1)(s+3)(s+4)[4(s+2)+12] \lambda^{s+2}-12(3 s+4)=0, \\
& \quad s \geqslant 3, \quad 0 \leqslant \lambda \leqslant 1 .
\end{aligned}
$$

As an example, let $s=3$, which yields $\lambda=0.6356$ and a population allocation ratio, $x^{*}=0.4625$. It is easy to show that this is a very good approximation, and that the approximations improve rapidly as $s$ increases.

\section{Summary of Sections 2 and 3}

In Section 2 it was assumed that each individual has the same amount of resources to invest, and the individual and population allocation ratios (which are identical) were presented as a function of the shapes of the male and female return functions. In Section 3 it was assumed that individuals have different amounts of resources to invest, and each individual adjusts its allocation ratio conditional on its available resources. The individual and population allocation ratios (which differ) were presented as functions of the shapes of the male and female return functions, and of the frequency distribution for the amount of resources available to each individual. These models make several assumptions that are unrealistic for many organisms. Models that are more flexible and realistic are presented in the next section.

\section{Fixfod Costs and Packaging Costs}

In many organisms individuals may have only partial control over their allocation ratios. Organisms with heterogametic sex chromosomes are obvious examples (reviewed by Bull, 1983), since the conception ratio is partly or completely a random process that can be approximately described by a binomial distribution (e.g., in vertebrates, see Williams, 1979). Monoecious and hermaphroditic plants are two further examples, since a 
plant may not be able to adjust the number of male flowers or flower parts, but may be able to vary allocation to each sex function. In each case a certain fixed amount of energy is allocated to both males and females, but these initial fixed investments are often too small for any returns to be realized without further investment. Heath (1977) has named initial investments in reproductive structures fixed costs for hermaphrodites. Here I adopt the additional phrase packaging costs for the minimum investment per offspring that cannot be reduced. Two examples of packaging costs are the cost of producing an egg in altricial birds, and the cost of producing a newborn in mammals, since in each case there are no reproductive returns without further investment. The important distinction between fixed and packaging costs is that the first is independent of the number of progeny produced (i.e., investment in reproductive structures), while the second increases lincarly with number of eggs (or fetuses, new-borns, etc.). Under this general distinction, fixed costs for reproductive structures apply equally well to dioecious and hermaphroditic organisms.

The models considered so far have examined total reproductive returns on total male or female investment per investment period. (The important problem of defining "investment period" is examined more carefully in the Discussion.) In many circumstances there is no loss of generality when ignoring both fixed and packaging costs (Charlesworth and Charlesworth, 1981). In some situations, however, considering these initial investments leads to some interesting insights, and a general treatment also leads to firmer conclusions about the robustness of the simpler models.

In this section I first present a general model that incorporates packaging costs, then examine a more realistic case designed with low fecundity, heterogametic organisms in mind; a description that fits many warmblooded vertebrates. Finally, a situation incorporating fixed costs is modeled after an organism with environmental sex determination (Bull, 1983), in which the costs of reproductive structures differ between males and females.

\subsection{General Model for Packaging Costs}

The approach taken here is an extension of Maynard Smith's (1980) work on sex allocation with a fixed conception ratio. Maynard Smith studied the situation in which (i) the shapes of the return curves on total investment differ between the sexes, (ii) the conception ratio is fixed at unity, (iii) a certain amount of investment in each offspring is mandatory, after which the parent can adjust its total allocation to each sex, and (iv) each individual in the population has the same amount of resources to invest. I simplify his results by using the methods devised above for describing shapes of return functions, and generalize the model by (a) allowing the 
amounts of resources available for reproduction to vary among individuals, (b) using the shapes of the return curves on investment per offspring, rather than on total investment, and (c) allowing the parent to adjust its allocation to each offspring, after the initial mandatory investment. Models considering variation in resources among individuals, and returns on adjustable investment per offspring rather than on total investment, are important extensions since these are common attributes of mammals, a group to which this sort of theory is often applied. My model also allows for the conception ratio to be different from unity. Interesting extensions not considered here include allowing the conception ratio to be stochastic, and allowing individuals to have different expected conception ratios according to the amount of resources available to them.

To begin, we need a more general form of Eq. (1), where $E_{i}$ is the expectation over $i$,

$$
w_{i}=\frac{\sum_{j=1}^{n_{m i}} f\left(x_{i j}+d\right)}{E_{i}\left[\sum_{j=1}^{n_{m i}} f\left(x_{i j}+d\right)\right]}+\frac{\sum_{j=1}^{n_{i j}} g\left(z_{i j}+d\right)}{E_{i}\left[\sum_{j=1}^{n_{i i}} g\left(z_{i j}+d\right)\right]},
$$

subject to the constraint that

$$
\sum_{j=1}^{n_{n i j}}\left(x_{i j}+d\right)+\sum_{j=i}^{n_{i j}}\left(z_{i j}+d\right)=k_{i}
$$

or, $x_{i}+z_{i}=k_{i}$, where

$w_{i}=$ expected reproductive success of $i$ th mother,

$k_{i}=$ total resources available to $i$ th mother,

$d=$ minimum investment per offspring,

$n_{m i}$ - number of male progeny by $i$ th mother,

$x_{i i}+d=$ investment in $j$ th son of $i$ th mother,

$n_{f i}=$ number of female progeny by $i$ th mother, $z_{i j}+d=$ investment in $j$ th daughter of $i$ th mother,

$x_{i}=\sum_{i=1}^{n_{\mathrm{m}}}\left(x_{i j}+d\right)$, the total investment in sons by the $i$ th mother,

$z_{i}=\sum_{i=1}^{n_{i i}}\left(z_{i j}+d\right)$, the total investment in daughters by the $i$ th mother.

Now assume that for given values of $x_{i}$ and $z_{i}$, a mother will always allocate additional resources to each offspring within a sex so as to maximize her total reproductive returns from that sex. For example, given 
a fixed value for $x_{i}$, she will choose values for the set $\left\{x_{i j}\right\}$ so as to maximize (or take the supremum over) $\sum_{j=1}^{n_{m i}} f\left(x_{i j}+d\right)$. In symbols,

$$
\begin{aligned}
& F\left(x_{i}\right)=\left\{\max \sum_{j=1}^{n_{m i}} f\left(x_{i j}+d\right) \mid x_{i}\right\} \\
& G\left(z_{i}\right)=\left\{\max \sum_{j=1}^{n_{f i}} g\left(z_{i j}+d\right) \mid z_{i}\right\}
\end{aligned}
$$

and

$$
w_{i}=\frac{F\left(x_{i}\right)}{E\left[F\left(x_{i}\right)\right]}+\frac{G\left(z_{i}\right)}{E\left[G\left(z_{i}\right)\right]} .
$$

With these definitions we can now find the values of $x_{i}$ and $z_{i}$ that maximize $w_{i}$ by the methods used to obtain (3), yielding

$$
\begin{gathered}
\frac{F^{\prime}\left(x_{i}^{*}\right)}{E\left[F\left(x_{i}^{*}\right)\right]}=\frac{G^{\prime}\left(z_{i}^{*}\right)}{E\left[G\left(z_{i}^{*}\right)\right]} \quad \text { for all } i \\
n_{m i} d \leqslant x_{i}^{*} \leqslant k_{i}-n_{f i} d \\
n_{f i} d \leqslant z_{i}^{*} \leqslant k_{i}-n_{m i} d \\
x_{i}^{*}+z_{i}^{*}=k_{i}
\end{gathered}
$$

which is analogous to (3), and the techniques for finding $x_{i}{ }^{*}$ and $z_{i}{ }^{*}$ from Sections 2 and 3 apply. The optimum allocations to individual offspring are the values $\left\{x_{i j}{ }^{*}\right\}$ and $\left\{z_{i j}{ }^{*}\right\}$ that satisfy (25), given $x_{i}=x_{i}{ }^{*}$ and $z_{i}=z_{i}{ }^{*}$. To find the values for males $\left\{x_{i j}{ }^{*}\right\}$, the method of LeGrangian mutipliers can be used

$$
\begin{gathered}
h_{i}=\sum_{j=1}^{n_{m i}} f\left(x_{i j}+d\right)-\Omega\left[\sum_{i=1}^{n_{m u}}\left(x_{i j}+d\right)-x_{i}^{*}\right]=0 \\
\frac{\partial h_{i}}{\partial x_{i j}}=f^{\prime}\left(x_{i j}{ }^{*}+d\right)-\Omega=0, \quad j=1,2,3, \ldots, n_{m i},
\end{gathered}
$$

solving,

$$
f^{\prime}\left(x_{i j}^{*}+d\right)=f^{\prime}\left(x_{i r}^{*}+d\right), \quad j \neq r, \quad j, r=1,2,3, \ldots, n_{m i}
$$

subject to the conditions

$$
\begin{gathered}
f^{\prime \prime}\left(x_{i j}{ }^{*}+d\right)<0 \\
\sum_{j=1}^{n_{m i}}\left(x_{i j}^{*}+d\right)=x_{i}^{*} .
\end{gathered}
$$


In order to simplify the development of the argument, let us assume as before that the female return function is linear (see the Appendix) after payment of the packaging cost, $d$, i.e., $g\left(z_{i j}+d\right)=z_{i j}$, and hence $G\left(z_{i}\right)=z_{i}-n_{f i} d$. There are two cases to examine. In the first, $f(d)=0$ and the shape of $f\left(x_{i j}+d\right)$ is such that $f^{\prime}$ is monotone decreasing (Figs. 5A-D). From (28), the values of $\left\{x_{i j}^{*}\right\}$ are all equal since $f$ is uniquely invertible, and $f^{\prime \prime}<0$, i.e., $x_{i j}{ }^{*}=x_{i r}{ }^{*}=\left(x_{i}{ }^{*} / n_{m i}\right)-d$ for all $j$ and $r$ (Figs. 5B-D). For the case in which $n_{m i}=1$ (Fig. 5A), and the fixed cost $d$ is the same for both males and females, the situation is identical to the model studied in Section 3 , where $k_{i}$ varies and the shape of the male return function is described by $t=1, s<1$. In this case large departures from a 1:1 population allocation ratio and strong individual-level biases are expected (see Sect. 3). When $d$ is small relative to $x_{i j}{ }^{*}$ (Fig. 5B), the return curve $F\left(x_{i}\right)$ quickly approaches linearity as $n_{m i}$ increases, thus resurrecting Fisher's (1930) equal investment theory for the population, and diminishing expected

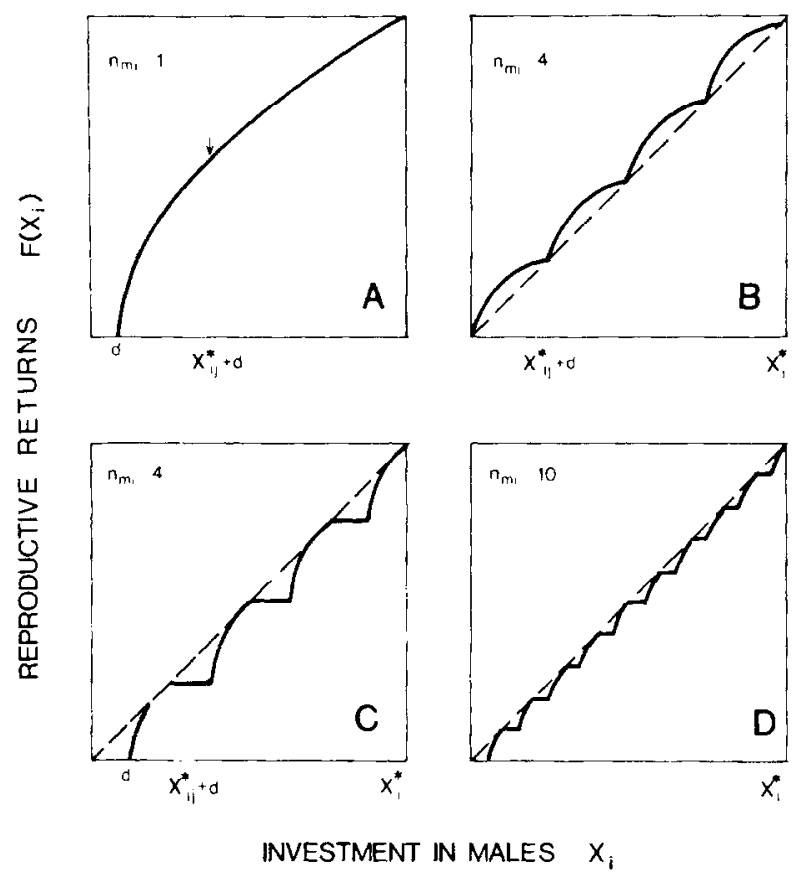

FiG. 5. Returns on male investment when there is a packaging cost, $d$, per offspring, and the shape of the returns for investment in each son is given by $s=\frac{1}{2}, t=1$ (Fig. 4); in $B$, $\left(d / x_{i j}{ }^{*}\right)=0$, while in $C$ and $D,\left(d / x_{i j}{ }^{*}\right)=1$. Note the rapid approach to linearity as the number of sons per investment period increases, and also that the cutoff point in $C$ and $D$ for further investment in each son is at the tangent where slope on further male investment equals slope on female investment. 
individual-level biases. When $d$ is a large portion of the investment in each offspring, the approach to linearity is slower (Fig. 5C), but by the time $n_{m i}=10($ Fig. 5D) the return curve is once again very close to linear. This entire argument is essentially the same when $f\left(x_{i j}+d\right)$ is $S$-shaped (Fig. 1C).

In summary, when the packaging costs are the same for males and females, and $n_{m i}=n_{f i}=1$, then the results of Section 3 apply, and when $n_{m i}$ and $n_{f i}$ are large, Fisher's equal investment result is extremely robust (see Discussion for exceptions). When $n_{m i}$ and $n_{f i}$ are greater than one, but still small, the problem is more difficult, but quite interesting since this is the situation for many mammals. This case is examined in the next section.

\subsection{An Example for Small Litters}

Consider the simple ease in which an organism produces a litter size of four, with $n_{m i}=n_{f i}=2$, and with a fairly long period of parental care. The initial cost of the litter is $4 d$. Assume that when $k_{i}<4 d$, a female fails to reproduce. Define $k_{i}^{\prime}=k_{i}-4 d$, and standardize $k_{i}^{\prime}$ so that $0<k_{i}^{\prime} \leqslant 1$ and $k_{i}=4 d$ corresponds to $k_{i}^{\prime}=0, x_{i}^{\prime}=x_{i}-2 d$ and $z_{i}^{\prime}=z_{i}-2 d$. so that $x_{i}^{\prime}$ and $z_{i}^{\prime}$ are male and female investments after packaging costs are paid, and $x_{i}^{\prime}+z_{i}^{\prime}=k_{i}^{\prime}$. The returns on investment for the two female littermates are assumed linear, $G\left(z_{i}^{\prime}\right)=z_{i}^{\prime}$, so there is no need to distinguish how energy is allocated between the two females. Let the returns on male investment for each male littermate be given by $f\left(x_{i j}\right)$, where $0 \leqslant x_{i j} \leqslant 0.5$, so that a mother with $k_{i}^{\prime}=1.0$ can have enough energy to invest the maximum of 1.0 in her two sons. Define $f\left(x_{i j}\right)$ by the beta CDF [see Eq. (4)] on the interval $[0,0.5]$, with $t=2, s=4$ (Fig. 6).

With these conditions, we can write the reproductive success $w_{i}$ of the $i$ th mother

$$
\begin{aligned}
& w_{i}^{\prime}\left(k_{i}^{\prime}\right)=\left[f\left(x_{i 1}\right)+f\left(x_{i 2}\right)\right]\left(\frac{E_{i}\left(z_{i}^{\prime}\right)}{2 E_{i j}\left[f\left(x_{i j}\right)\right]}\right)+z_{i}^{\prime} \\
& x_{i}^{\prime}+z_{i}^{\prime}=k_{i}^{\prime} .
\end{aligned}
$$

There are five regions that need to be considered (Fig. 6), and five strategies of allocation depending on the resources $k_{i}^{\prime}$ available
(i) $k_{i}^{\prime}<\lambda$
$x_{i 1}=0 \quad x_{i 2}=0 ; \quad z_{i}^{\prime}=k_{i}^{\prime}$
(ii) $\lambda<k_{i}^{\prime}<\delta$
$x_{i 1}=k_{i}^{\prime} ; \quad x_{i 2}=0 ; \quad z_{i}^{\prime}=0$
(iii) $\delta<k_{i}^{\prime}<\gamma$
$x_{i 1}=\delta ; \quad x_{i 2}=0 ; \quad z_{i}^{\prime}=k_{i}^{\prime}-\delta$
(iv) $\gamma<k_{i}^{\prime}<2 \delta$
$x_{i 1}=k_{i}^{\prime} / 2 \quad x_{i 2}=k_{i}^{\prime} / 2 ; \quad z_{i}^{\prime}=0$
(v) $2 \delta<k_{i}^{\prime}<1.0$
$x_{i 1}=\delta$
$x_{i 2}=\delta$
$z_{i}^{\prime}=k_{i}^{\prime}-2 \delta$, 


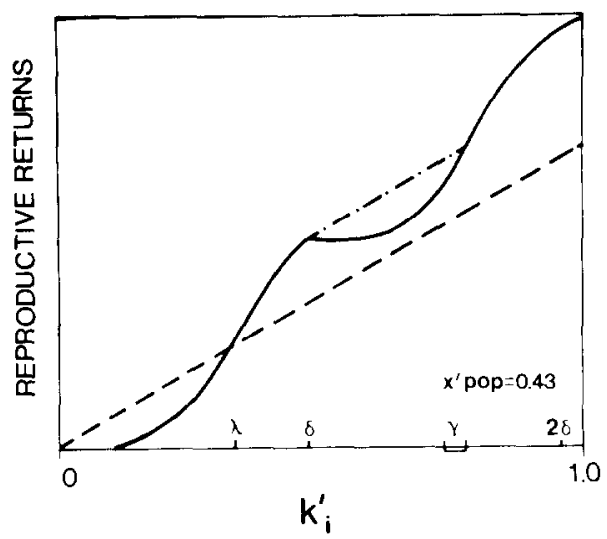

Fia. 6. An example when broods are composed of two sons and two daughters, with parameters $a=2 . b=6 ; s=4, t=2$. Families that have resources, $k_{i}^{\prime}$, less than $i_{n}$ will invest entirely in daughters (returns shown in dashed line). When $i<k_{i}^{\prime}<\delta$, investment only in the first son yiclds the greatest reproductive success (solid curve). When $\delta<k_{;}^{\prime}<\gamma$, investing $\delta$ in the first son and $k_{i}^{\prime}-\dot{\delta}$ in daughters is the best reproductive strategy (returns shown in dotdash line). When $i<k_{i}^{\prime}<2 \delta$, investing $k_{i j}^{\prime} 2$ in each son. and nothing in daughters is the best strategy (see text). Finally, when $k_{i}^{\prime}>2 \delta$, greatest returns are achieved by investing $\delta$ in each son, and $k_{i}^{\prime}-2 \delta$ in daughters.

At equilibrium we have the following conditions,

(vi) $f(\lambda)(r / q)=i$

(vii) $\left(\left.\frac{\partial f\left(x_{i j}\right)}{\partial x_{i j}}\right|_{x_{i t}=\delta}\right)(r / q)=1$

(viii) $2 f(\gamma / 2)(r / q)=f(\delta)(r / q)-\delta+\gamma$

$$
r / q=\frac{E_{i}\left(z_{i}^{\prime}\right)}{2 E_{i j}\left[f\left(x_{i j}\right)\right]}
$$

The values of $E_{i}\left(z_{i}^{\prime}\right)$ and $2 E_{i j}\left[f\left(x_{i j}^{\prime}\right)\right]$ can be obtained as functions of $\lambda, \delta$, and $\gamma$ from conditions (i)-(v), given the PDF of $k_{i}^{\prime}, h\left(k_{i}^{\prime}\right)$. Assume $h\left(k_{i}^{\prime}\right)$ is a beta PDF, as in Eq. (14). Conditions (vi)-(viii) provide three independent equations in the three unknowns $\lambda, \delta$, and $\gamma$.

An example is shown in Fig. 6 . The distribution of resources $h\left(k_{i}^{\prime}\right)$ is described by a beta PDF with parameters $a=2$ and $b=6$; a distribution with a strong right skew and a mode at $\frac{1}{6}$ (cf. Fig. 3). The shape for returns on investment in each son is $f\left(x_{i j}\right)$, which is described in this example by a beta CDF with parameters $s=4$ and $t=2$ (see Fig. 6). Conditions (i)-(v) describe the allocation strategies as a function of $k_{i}^{\prime}$, and the returns are given by the ordinate with the greatest height for each value of $k_{i}^{\prime}$ in Fig. 6. 
To obtain the equilibrium values of $\lambda, \delta$, and $\gamma$, condition (vi) states that at the smallest value of $k_{i}^{\prime}$, called $\lambda$, where the curves cross, the returns on pure female investment, $\lambda$, equal the returns on pure male investment, $f(\lambda)(r / q)$ [see Eq. (29)]. As $k_{i}^{\prime}$ increases past $\lambda$, the slope decreases for the returns on pure male investment, $\left.\left[\partial f\left(x_{i j}\right) / \partial x_{i j}\right)\right](r / q)$, until further investment in the first son gives returns at a slope of less than one, as described by condition (vii). This point is called $\delta$. As $k_{i}^{\prime}$ increases above $\delta$, allocating $\delta$ to the first son and $k_{i}^{\prime}-\delta$ to daughters gives the greatest returns (dot-dash line). The dot-dash line crosses the second half of the pure male investment returns curve just before $\lambda+\delta$. The intersection occurs before $\lambda+\delta$ since the returns on pure male investment are greatest when investing $k_{i}^{\prime} / 2$ in each son [see Eq. (28)], rather than $\delta$ in the first and $k_{i}^{\prime}-\delta$ in the second as shown in Fig. 6. Condition (viii) describes this intersection at $k_{i}^{\prime}-\gamma^{\prime}$

The results for this example are $\lambda=0.34, \delta=0.48, \gamma=0.77$. Using these values and the distribution for $h\left(k_{i}^{\prime}\right)$, the population allocation ratio $x_{\mathrm{pop}}^{\prime}$ is 0.43. All of these values, including $x_{\text {pop }}^{\prime}$, are independent of the magnitude of the packaging costs, $d$. But the magnitude of $d$ plays two roles. First, $x_{\text {pop }}^{\prime}$ is not a true measure of the population allocation ratio, since it ignores initial costs. The true value, $x_{\mathrm{pop}}$, is given by $x_{\mathrm{pop}}=\left(x_{\mathrm{pop}}^{\prime}+n_{m i} d\right) /$ $\left[1+\left(n_{m i}+n_{f i}\right) d\right]$, and taking care to standardize $d$ in the same way as $x^{\prime}$ has been standardized. When $d$ is five percent of the maximum value of $k_{i}$ in the population, then $x_{\mathrm{pop}}$ is 0.44 , or only slightly closer to 0.5 . The second role of $d$ is more important for applications, since the value of $d$ sets the minimum amount of energy required to reproduce. and determines what proportion of the resources available can be allocated to either sons or daughters, and what proportion is set by initial costs. Values of $\lambda, \delta, \gamma$, and $x_{\text {pop }}^{\prime}$ are presented as functions of the shapes of $h\left(k_{i}^{\prime}\right)$ and $f\left(x_{i j}\right)$ in Figs. 7A, B.

\subsection{A Fixed Costs Model}

The assumptions for this section are that sex (gender) is environmentally determined (ESD; Bull, 1983), and that costs of reproductive structures differ between males and females. Imagine a species with ESD in which females brood eggs in brooding chambers, and the costs of these chambers is independent of number of eggs produced. Plausible examples might include plants with a small, fixed number of fruits, or many fish that brood their eggs.

For an individual to reproduce successfully as a female, it must first invest in female reproductive structures (fruits, brood chambers, etc.) which $\operatorname{cost} d_{f}$, and the remaining energy $k_{i}-d_{f}$ is invested in offspring. Reproductive returns are zero when $k_{i} \leqslant d_{f}$, and returns are $k_{i}-d_{f}$ when this value is greater than zero (i.e., returns are assumed to be linear after the fixed cost 

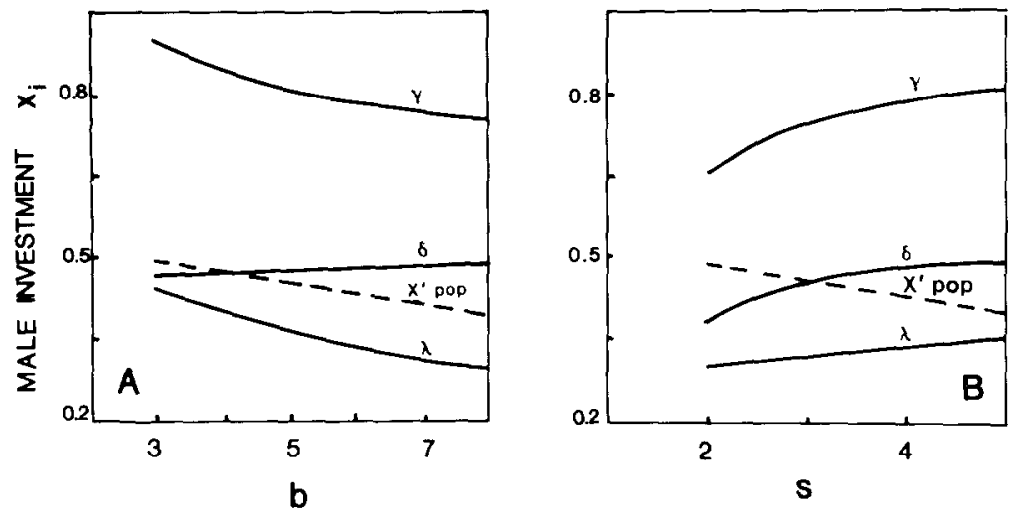

FIG. 7. Exploration of the parameter space for the example in which broods consist of two sons and two daughters. $A$, the distribution of resources, $k_{i}^{\prime}$, is varied, with parameters $a=2$; $s=4, t=2$. $B$, the shape of the male return curve is varied, with $a=2, b=6 ; t=2$.

is paid). Likewise for males, the fixed cost is $d_{m}$, and returns are $k_{i}-d_{m}$. Assume that the female reproductive structures are more costly than the male reproductive structures, i.e, $d_{f}>d_{m}$. (Since the results are symmetric, this assumption can easily be reversed.) A general picture of this situation is presented in Fig. 8A. When the ith individual has small amounts of resources available for reproduction, it will be favored to reproduce as a male $\left(x_{i}=1\right)$; when it has large amounts of resources, it will be favored to reproduce as a female $\left(x_{i}=0\right)$; and at some point, $\lambda$, returns on male and female investment will be equal.

In general, the fitness of the $i$ th individual in the population, $w_{i}$, as a function of its resources available for reproduction, $k_{i}$, and its sex, $x_{i}$, is

$$
w_{i}\left(k_{i} x_{i}\right)= \begin{cases}0, & 0<k_{i} \leqslant d_{m} \\ \left(k_{i}-d_{m}\right)\left(\frac{\left.E\left[k_{i}-d_{f}\right)\left(1-x_{i}\right)\right]}{E\left[\left(k_{i}-d_{m}\right) x_{i}\right]}\right), & d_{m}<k_{i}<\lambda \\ k_{i}-d_{f}, & \lambda<k_{i} \leqslant 1 .\end{cases}
$$

If we specify the distribution of resources in the population as $h\left(k_{i}\right)$, then

$$
\begin{aligned}
E\left[\left(k_{i}-d_{f}\right)\left(1-x_{i}\right)\right] & =\int_{i}^{l}\left(k_{i}-d_{f}\right) h\left(k_{i}\right) d k_{i} \\
E\left[\left(k_{i}-d_{m}\right) x_{i}\right] & =\int_{d_{m}}^{\lambda_{m}}\left(k_{i}-d_{m}\right) h\left(k_{i}\right) d k_{i} .
\end{aligned}
$$

To illustrate, let $h(k$,$) be a beta PDF with parameters a$ and $b$, as in (14). An example with specific parameter values is shown in Fig. 8A, and part of the parameter space is explored in Fig. 8B. In this case $x_{\text {pop }}$ always includes 

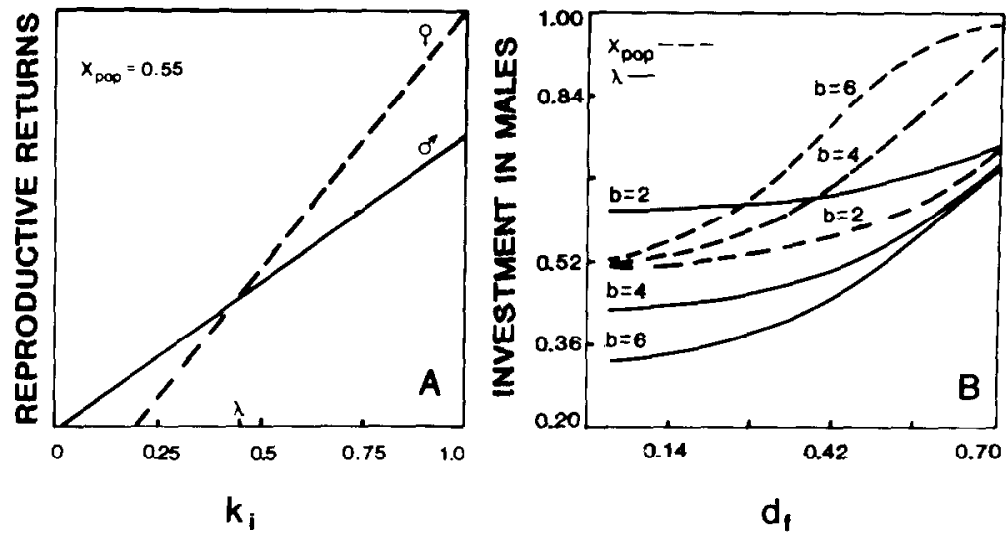

FiG. 8. Fixed costs of reproductive structures differ between the sexes. $A$, a specific example, with $d_{m}=0.02, d_{f}=0.20, a=2, b=4$, yielding $\lambda=0.45$ and $x_{p o p}=0.55$; and $B$, an exploration of the parameter space, with $d_{m}=0.02$ and $a=2$.

the fixed costs, $d_{m}$ and $d_{f}$, since they are unequal and often represent a large fraction of the investment. The most interesting result for the cases studied here is that when $d_{f}>d_{n}, x_{\text {pop }}$ is male biased. The magnitude of the population bias depends on the difference $d_{f}-d_{m}$, i.e, the extent to which the return functions differ for the two sexes.

It would be interesting to know if a male bias in $x_{\text {pop }}$ always results when $d_{f}>d_{m}$. From (32) we can see that when $k_{i}=\lambda, \lambda-d_{f}=$ $\left(\lambda-d_{m}\right) E(g) / E(f)$, and since $d_{f}>d_{m}$, it follows that $E(g)<E(f)$. This means that the resources excluding fixed costs allocated to males by the population, $\mathrm{E}(f)$, is greater than the total resources excluding fixed costs allocated to females, $E(g)$. Note that this argument requires that both $f$ and $g$ be linear, in order for $E(g)$ and $E(f)$ to reflect accurately the population allocations. Whether this male bias always holds when fixed costs are included, or when either $f$ or $g$ are nonlinear, is not presently known. (I thank P. D. Taylor for this particular result about the generality of male bias when $d_{f}>d_{m}$.)

It has been assumed here that the size of the brood chamber does not limit the reproductive potcntial of a femalc. Heath (1977) has proposed that a limit in the capacity of brood chambers may explain the distribution of hermaphroditism, since such a fixed limit may make it profitable for an individual to pay the extra fixed costs of male structures and simultaneously reproduce as a male. He suggests that this may explain the observed association between brooding and hermaphroditism (see also Ghiselin, 1969). Since the model presented here does not consider limits on female reproductive potential, it is not directly applicable to Heath's ideas, but could easily be extended to incorporate them. See Charnov (1982) for models of this sort. 


\section{Discussion}

\subsection{Defining an Investment Period}

For all models developed in this paper, the reproductive returns on investment are for a single investment period. An investment period is the smallest time interval during which patterns of allocation have no effect on relative returns in other intervals; in other words, the interval during which an individual's amount and ratio of allocation has no effect on the difference between the sexes in potential reproductive returns during other time intervals. Consider some examples.

(i) A parasitic hymenopteran lays one egg on each suitable host that it encounters. Whether the egg develops into a male or a female on onc host has no effect on the reproductive returns of an offspring laid on another host. The investment period is a single egg-laying bout (cf. Green, 1982), and the energy content of the host is the resource available for that investment period. Thus an egg-laying sequence is a series of independent investment periods.

(ii) High fecundity organisms invest little in each offspring relative to total investment. Examples are fish, insects, and marine invertebrates that lay large clutches of eggs, and do not give parental care. The number of eggs (total investment) and ratio of males to females in the clutch has no effect on the potential difference in returns between male and female investment in future clutches. Thus each clutch of eggs can be treated independently.

(iii) Human females produce one or two offspring at a time over about a 20 -yr period. The period of parental investment is long, and it is common for parents to pass resources to adult offspring (e.g., inheritance, dowry, brideprice, etc.). Parents may be viewed as having a nearly fixed amount of resources to divide among their lifetime brood. What is given to one offspring directly reduces the amount of resources available for the others.

(iv) Must large mammals produce a small number of offspring at one time, and the overlap between parental care for one brood and the beginning of the next brood is usually short. However, there are a number of cases in which it is clear that one sex is more costly to rear than the other (Clutton-Brock et al., 1981; Clutton-Brock and Albon, 1982). One example is the greater frequency of skipping the following breeding season after rearing a male offspring in red deer (Clutton-Brock et al., 1981). A complementary example is the longer period until the next birth after rearing a female child in some primates. In these species daughters inherit maternal status in the local band, and often receive high levels of maternal 
investment, while males disperse after weaning, and often receive lower levels of maternal investment (data reviewed in Hinde, 1983). In red deer and these primates there is a negative correlation between the resources expended in one period and the resources available in the next. However, the correlation is weaker than in the human case, where resources given to one offspring are directly taken away from the total resources available for others. So, this situation is best characterized by the transition probabilities describing the amount of resources available and amount expended in one period, and the resources available for future breeding periods. The different methods for analyzing these four types of investment periods are discussed in the following section.

\subsection{Robustness of Equal Allocation}

When returns on investment differ between the sexes, Fisher's (1930) equal allocation theory does not hold. To analyze a particular situation, we need to consider (a) the length of an investment period, (b) the male and female return curves per offspring, (c) the number of offspring in an investment period, and (d) the shape of the resource distribution curve. The strong effect that the shape of the resource distribution curve has on individual and population patterns was clearly illustrated in Figs. 4, 7, and 8 , and will not be discussed further. The potential magnitude of this effect has not been appreciated before, since it is only apparent within a general quantitative approach.

Competition among male relatives, among female relatives, and inbreeding also affect the allocation ratio by setting the shapes of the return curves, but these effects will not be discussed here since this theory was recently synthesized and the literature reviewed (Taylor, 1981; Charnov, 1982; Nunney, 1985; Frank, 1985, 1986a, b). Also, it is assumed here that control of the sex ratio is by autosomal genes with additive effects only; or equivalently, that the "realized" level of selection is the individual (Hamilton, 1967; Charnov, 1982; Maynard Smith, 1982; Frank, 1983).

(i) Sequential independent periods of investment and one offspring per period. Here all that must be considered are the return curves on investment for a single offspring of each sex, and the models of Section 3 (Fig. 4) apply. This situation will often be associated with extreme individual- and population-level biases away from 1:1, since increasing numbers of offspring per period make the return curves more nearly linear (Sect. 5).

(ii) Sequential independent periods of investment and many offspring per period. The returns for both males and females will be very close to linear because of the "smoothing" effect of large clutch sizes (Figs. 5A-D). If the fixed and packaging costs are the same for both sexes, and there is no 
inbreeding or within-sex competition among relatives, then equal allocation is a very robust prediction, and little individual-level variation is expected. If the fixed or packaging costs differ between the sexes, or clutch size is small, then the models of Section 5 (Figs. 8A, B) apply.

(iii) Sequential hroods and fixed amount of resources for lifetime investment. The results for this case depend on lifetime brood size. If the lifetime brood is one, then the models for (i) above apply. If the lifetime brood is large, then the models for (ii) above apply. When the lifetime brood size is small, the situation is complex, and both individual- and population-level biases are expected (Sect. 5, Figs. 6.7). The extent of these biases will be less than for single-offspring broods, since multiple offspring tend to reduce nonlinearities (cf. Figs. 4B and 6).

(iv) Sequential broods and a negative correlation among amounts of resources available in each period, described by transition probabilities between amounts available and invested in one period and amount available for the next. Many organisms fall into this category, for which there is no quantitative theory. The Trivers and Willard (1973) hypothesis is a qualitative prediction suggesting that the sex ratio at birth produced by an individual should be skewed in favor of the sex with greater reproductive returns. For example, in a polygynous species a mother in poor condition is predicted to have more daughters, and a mother in good condition more sons. There are data from some specics consistent with this hypothesis (Trivers and Willard, 1973), although the existence and ubiquity of a labile birth sex ratio in vertebrates has been questioned (Myers, 1978; Williams, 1979). Nearly all of the species that have been examined to test the Trivers-Willard hypothesis are of the sort that have sequential broods and a negative correlation in resources expended in one period and those available in the next, these species being mostly ungulates and primates. Attempts have been made to compare the population allocation ratios in these species to some quantitative predictions, such as Fisher's (1930) equal allocation theory, but no firm conclusions have been reached (e.g., Clutton-Brock et al., 1981; Clutton-Brock and Albon, 1982). The results from the present paper suggest that these sorts of comparisons are unsatisfactory, since no adequate quantitative theory exists for population allocation in these groups.

\section{APPENDIX: Nonlinear Returns for Both Sexes}

The models in this paper all assume linearity for returns on investment in one sex, and nonlinearity for the other sex. Here I provide an example of how to approach a problem in which returns are nonlinear for both sexes. 
In the simplest case, all individuals have the same amount of energy to invest, and at equilibrium all invest $x^{*}$ in males and $1-x^{*}$ in females. The equilibrium for this case was shown to be [Eq. (3)]

$$
\frac{f^{\prime}\left(x^{*}\right)}{f\left(x^{*}\right)}=\frac{g^{\prime}\left(1-x^{*}\right)}{g\left(1-x^{*}\right)}
$$

If $f\left(x^{*}\right)$ is a beta CDF with parameters $s_{m}$ and $t_{m}$, and $g\left(1-x^{*}\right)$ is a beta CDF with parameters $s_{f}$ and $t_{f}$, then at equilibrium

$$
\frac{(x)^{s_{m}-1}(1-x)^{t_{m}-1}}{\int_{0}^{x}(q)^{s_{m}-1}(1-q)^{t_{m}-1} d q}=\frac{(1-x)^{s_{t}-1}(x)^{t_{f}-1}}{\int_{0}^{1}{ }^{x}(r)^{s_{f}-1}(1-r)^{t_{f}-1} d r}
$$

or, rcarranging

$$
(x)^{s_{m}}{ }^{t}(1-x)^{t_{m}} \quad s_{f}=\frac{\int_{0}^{x}(q)^{s_{m}-1}(1-q)^{t_{m}} \quad d q}{\int_{0}^{1} r(r)^{s_{t}}{ }^{1}(1-r)^{t^{\prime \prime}-1} d r} .
$$

This equation can easily be solved by standard numerical techniques. The same general approach can be applied to the models of Sections 3 and 5.

\section{ACKNOWLEDGMENTS}

Discussions with Bobbi Low contributed to the ideas presented in this paper, and Richard Alexander, Peter Smouse, and Peter Taylor gave helpful comments on earlier drafts of the manuscript.

\section{REFERENCES}

BuLl, J. J. 1981. Sex ratio evolution when fitness varies, Heredity, 46, 9-26.

BulL, J. J. 1983. "The Evolution of Sex Determining Mechanisms." Benjamin-Cummings, Menlo Park, Calif.

Charlesworth, D., and Charlesworth, B. 1981. Allocation of resources to male and female function in hermaphrodites, Biol. J. Linn. Soc. 15, 57-74.

Charnov, E. L. 1979a. Simultaneous hermaphroditism and sexual selection, Proc. Natl. Acad. Sci. U.S.A. 76, 2480-2484

Charnov, E. L. 1979b. The genetical evolution of patterns of sexuality: Darwinian fitness, Amer. Nat. 113, 715-734.

Cirarnov, E. L. 1982. "The Theory of Sex Allocation," Princeton Univ. Press, Princeton, N. J.

Charnov, E. L., Los-den Hartogh, R. L., Jones, W. T., and van den Assem. J. 1981. Sex ratio evolution in a variable environment, Nature (London) 289, 27-33.

Charnov, E. L., Maynard Smith, J., and Bull, J. J. 1976. Why be an hermaphrodite? Nature (London) 263, 125-126. 
Clark, A. B. 1978. Sex ratio and local resource competition in a prosimian primate, Science (Washington, D. C.) 201, 163-165.

Clutton-Brock, T. H., Albon, S. D, and Guinness, F.E. 1981. Parental investment in male and female offspring in polygynous mammals, Nature (London) 289, 487-489.

Clutton-Brock, T. H., and Albon, S. D. 1982. Parental investment in male and female offspring in mammals, in "Current Problems in Sociobiology," pp. 223-248. Cambridge Univ. Press. Cambridge.

Eshel, I., and Feldman, M. W. 1982a. On evolutionary genetic stability of the sex ratio, Theor. Pop. Biol. 21, 430-439.

Eshel, I., AND Feldman, M. W. 1982b. On the evolution of sex determination and the sex ratio in haplodiploid populations, Theor. Pop. Biol. 21, $440-450$.

Fisht:R, R. A. 1930. "The Genetical Theory of Natural Selection," Oxford Univ. Press (Clarendon), Oxford.

Frank, S. A. 1983. A hierarchical view of sex-ratio patterns, Fl. Entomol. 66, 42-75.

Frank, S. A. 1985. Hierarchical selection theory and sex ratios. II. On applying the theory, and a test with fig wasps, Evolution 39, 949-964.

Frank, S. A. 1986a. Hierarchical selection theory and sex ratios. I. General solutions for structured populations, Theor. Pop. Biol. 29. 312-342.

Frank. S. A. 1986b. The genetic value of sons and daughters, Heredity 56, 351-354.

Ghistu.IN, M. T. 1969. The evolution of hemaphroditism annong animals, Quart. Ret. Biol. 44. 189-208.

Grafen, A. 1984. Natural selection, kin selection and group selection, in "Behavioural Ecology" (J. R. Krebs and N. B. Davies. Eds.). pp. 62-86. Sinauer, Sunderland. Mass.

Grein. R. F. 1982. Optimal foraging and sex ratio in parasitic wasps, J. Theor. Biol. 95. 43-48.

Hamiltox. W. D. 1967. Extraordinary sex ratios, Science (Washington, D. C.) 156, 477-488.

HEATH, D. J. 1977. Simultaneous hermaphroditism: Cost and benefit, J. Theor. Biol. 64, 363-373.

HiNibF, R. A. 1983. "Primate Social Relationships: An Integrated Approach," Blackwell, Oxford.

MacArthli, R. H. 1965. Ecological consequences of natural selection, in "Theoretical and Mathematical Ecology" (T. H. Waterman and H. Morowitz, Eds.), pp. 388-397, Blaisdell, New York.

Maynard Smith. J. 1980. A new theory of sexual investment, Behar. Ecol. Sociobiol. 7. 247-251.

Maynard Smith, J. 1982. "Evolution and the Theory of Games," Cambridge Univ. Press. Cambridge.

Myers, J. H. 1978. Sex-ratio adjustment under food stress: maximization of quality or numbers of offspring, Amer. Nat. 112, 381-388.

Nunver, L. 1985. Female-biased sex ratios: Individual or group selection? Evolution 39, $349-361$.

Shaw, R. F., ANd Mohler, J. D. 1953. The selective advantage of the sex ratio, Amer. Nat. 87, 337-342.

TAYLOR, P. D. 1981. Intra-sex and inter-sex interactions as sex determinants, Nature (London) 291, 64-66.

TAYLOR, P. D. 1985. A general mathematical model for sex allocation, $J$. Theor. Biol. 112, $799-818$.

Trivers, R. L., and WiLlard, D. E. 1973. Natural selection of parental ability to vary the sex ratio, Science (Washington, D. C.) 179, 90-92.

Uyenoyama, M. K., and Bengtsson, B. O. 1979. Towards a genetic theory for the evolution of the sex ratio, Genetics $93,721-736$. 
Uyenoyama, M. K.. And Bengtsson, B. O. 1981. Towards a genetic theory for the evolution of the sex rato. II. Haplodiploid and diploid models with sibling and parental control of the brood sex ratio and brood size, Theor. Pop. Biol. 20, 57-79.

Uyenoyama, M. K., AND Bengtsson, B. O. 1982. Towards a genetic theory for the evolution of the sex ratio III. Parental and sibling control of brood investment ratio under partial sib-mating, Theor. Pop. Biol. 22, 43-68.

Williams, G. C. 1979. The question of adaptive sex ratio in outcrossed vertebrates, Proc. Roy. Soc. London Ser. B 205, 567-580. 